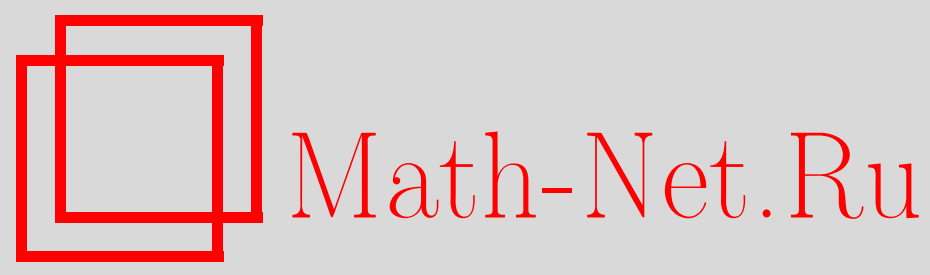

Л. М. Самойлов, Замечание о трехчленных тождествах в ассоциативных алгебрах, Матем. заметки, 1999, том 65, выпуск $2,254-260$

DOI: https://doi.org/10.4213/mzm1047

Использование Общероссийского математического портала Math-Net.Ru подразумевает, что вы прочитали и согласны с пользовательским соглашением http://www . mathnet.ru/rus/agreement

Параметры загрузки:

IP : 54.197 .217 .227

26 апреля 2023 г., 14:15:51

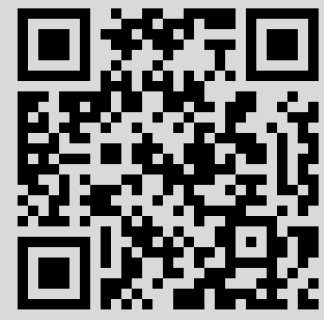




\title{
ЗАМЕЧАНИЕ О ТРЕХЧЛЕННЫХ ТОЖДЕСТВАХ В АССОЦИАТИВНЫХ АЛГЕБРАХ
}

\section{Л. М. Самойлов}

\begin{abstract}
В работе рассматриваются трехчленные тождества ассоциативных алгебр (в общем случае без единицы) над бесконечньп полем, т.е. тождества вида $\alpha m_{1}+\beta m_{2}+\gamma m_{3}=0$, где $\alpha, \beta, \gamma-$ скаляры, $m_{1}, m_{2}, m_{3}$ - различные мономы. Показано, что каждое такое нетривиальное тождество влечет полугрупповое тождество. Алгебры с трехчленными тождествами охарактеризованы на язьке многообразий.

Библиография: 3 названия.
\end{abstract}

В работе предполагается, что основное поле $F$ является бесконечньм и все алгебры являются ассоциативными $F$-алгебрами.

В ряде работ (см., например, [1], [2]) исследовались так называемые полугрупповые тождества, т.е. полиоднородные тождества, имеюшие вид $m_{1}=m_{2}$, где $m_{1}$ и $m_{2}-$ различные мономы. Для таких тождеств была установлена тесная взаимосвязь с тождеством энгелевости. В дальнейшем тождество энгелевости степени $n$ будем обозначать через $E_{n}$ :

$$
E_{n}=[x, \underbrace{y, \ldots, y}_{n}] .
$$

Произвольньй полиоднородньй полином вида $\sum_{i=1}^{n} \alpha_{i} m_{i}$, где $\alpha_{i} \in F, \alpha_{i} \neq 0$, a $m_{i}-$ различные мономы, называется приведенным слева (справа), если $n>1$ и наибольшее общее начало (конец) мономов $m_{i}$ является пустым. Если полином приведен и слева, и справа, то он называется приведенным.

В настоящей работе исследуются трехчленные тождества, т.е. полиоднородные тождества вида $\alpha m_{1}+\beta m_{2}+\gamma m_{3}=0$, где $\alpha, \beta, \gamma \in F, m_{1}, m_{2}, m_{3}$ - различные мономы и хотя бы два коэффициента отличны от 0 . Для тождеств такого вида получены результаты, обобщающие соответствующие результаты о полугрупповых тождествах. Мы также охарактеризуем алгебры с трехчленными тождествами на языке многообразий. Центральное место в нашем рассмотрении будут занимать тождества, аналогичные тождеству энгелевости.

В работе доказана следующая

ТЕоремА. Для алгебры $A$ (в общем случае без единииы) над бесконечным полем следующие условия әквивалентны:

1) алгебра А удовлетворяет трехчленному тожсдству (приведенному, приведенному слева, приведенному справа); 
2) для некоторых $m, n, k$ алгебра $A$ удовлетворяет тождеству $y^{m} E_{n} y^{k}=0$ $\left(E_{n}=0, E_{n} y^{k}=0, y^{m} E_{n}=0\right)$;

3) в алгебре $A$ выполнено полугрупповое тохдество (приведенное, приведенное слева, приведенное справа);

4) многообразие $\operatorname{Var}(A)$ не содержит алгебру верхнетреугольных матрич, второго порядка $\Lambda_{2}$.

Из этой теоремы следует, что изучение трехчленных тождеств над бесконечным полем полностью сводится к изучению полугрупповых тождеств. Заметим также, что если тождество $m=0$, где $m$ - моном, считать приведенньм (и трехчленным), то теорема очевидно верна.

Лемма 1. Пусть алгебра А над произвольным полем удовлетворяет полиоднородному трехчленному тохдеству с нулевой суммой коэффичиентов (приведенному, приведенному слева, приведенному справа). Тогда алгебра А удовлетворяет полиоднородному трехчленному тохсеству (приведенному, приведенному слева, приведенному справа) той же степени, но от двух переменных.

ДокАЗАТЕЛЬСТво. Пусть $f=\alpha m_{1}+\beta m_{2}+\gamma m_{3}-$ трехчленное тождество от переменных $x_{1}, \ldots, x_{n}$, выполняющееся в алгебре $A$.

Допустим, что один из коэффициентов $\alpha, \beta, \gamma$, для определенности $\gamma$, равен 0 , а два других отличны от 0 . Пусть моном $m_{0}$ является наибольшим общим началом, а моном $m_{4}$ - наибольшим обшим конщом мономов $m_{1}$ и $m_{2}$ (в случае левой приведенности $m_{0}-$ пустой моном, а в случае правой приведенности $m_{4}$ - пустой моном). Представим тождество $f=0$ в виде

$$
\alpha m_{0} x_{i} m_{1}^{\prime} x_{k} m_{4}+\beta m_{0} x_{j} m_{2}^{\prime} x_{l} m_{4}=0,
$$

где $m_{1}^{\prime}$ и $m_{2}^{\prime}$ - мономы (возможно пустые), $i \neq j, k \neq l$. Подставим в полином $f$ $x$ вместо $x_{i}$ и $y$ вместо $x_{j}$. Остальные переменные определим следующим образом. Если $\{i, j\}=\{k, l\}$, то положим их равными $y$. Если $\{i, j\} \cap\{k, l\}=\varnothing$, то положим $x_{k}=x, x_{l}=y$. Если $k=i$ или $k=j$, но $l \notin\{i, j\}$, то положим $x_{l}=y$ или $x_{l}=x$ соответственно. Аналогично, если $l=i$ или $l=j$, но $k \notin\{i, j\}$, то положим $x_{k}=y$ или $x_{k}=x$ соответственно. Все остальные переменные положим равными $y$. Очевидно, что при такой подстановке переменных в тождество $f=0$ получается полиоднородное двучленное тождество от двух переменных, которое будет приведенным (приведенньп слева, приведенным справа), если таковым было тождество $f=0$.

Осталось рассмотреть случай, когда все коэффициенты $\alpha, \beta, \gamma$ ненулевые. Допустим, что моном $m_{0}$ является наибольшим общим началом, а моном $m_{4}$ - наибольшим общим концом мономов $m_{1}, m_{2}$ и $m_{3}$ (в случае левой приведенности $m_{0}$ - пустой моном, а в случае правой приведенности $m_{4}-$ пустой моном). Тождество $f=0$ можно записать в виде

$$
\alpha m_{0} x_{i} m_{1}^{\prime} x_{a} m_{4}+\beta m_{0} x_{j} m_{2}^{\prime} x_{b} m_{4}+\gamma m_{0} x_{k} m_{3}^{\prime} x_{c} m_{4}=0,
$$

г де $m_{1}^{\prime}, m_{2}^{\prime}, m_{3}^{\prime}$ - мономы (возможно пустые), а $x_{i}, x_{j}, x_{k}, x_{a}, x_{b}, x_{c}$-переменные. В силу выбора $m_{0}$ и $m_{4}$ равенства $i=j=k$ и $a=b=c$ невозможны. Для определенности будем считать, что $i \neq j, i \neq k$. Положим $x_{i}=x, x_{j}=y, x_{k}=y$. Если $\{i, j, k\}=\{a, b, c\}$, то остальные переменные положим равными $y$. Если $\{i, j, k\} \cap\{a, b, c\}=\varnothing$, то положим одну из переменных $x_{a}, x_{b}, x_{c}$ равной $x$, а остальные переменные положим равными $y$. Если же хотя бы одна из переменных $x_{a}, x_{b}, x_{c}$ не принадлежит множеству $\left\{x_{i}, x_{j}, x_{k}\right\}$, 
то ее выбор осуществим так, чтобы при подстановке в полином $f$ на месте переменных $x_{a}, x_{b}, x_{c}$ стояли как $x$, так и $y$. Остальные переменные снова положим равными $y$. Так как $\alpha+\beta+\gamma=0$, в результате такой подстановки переменных в тождество $f=0$ получается полиоднородное трехчленное тождество от двух переменных, которое будет приведенным (приведенным слева, приведенньг справа), если таковьм было тождество $f=0$. Лемма доказана.

ЛЕмма 2. Пусть полиоднородный полином $f$ от двух переменных $x$ и у имеет нулевую сумму коэффичиентов и представи́м в виде

$$
f=\alpha m_{0} x^{k} y u+m_{0} y g \quad\left(f=\alpha u y x^{k} m_{0}+g y m_{0}\right)
$$

әде $\alpha \in F, \alpha \neq 0, m_{0} u и$-мономы (возмохсно пустые), $g$ - полином от $x u y$, $k \geqslant 1$. Тогда однородная степени 1 по $x$ компонента полинома $f(x+y, y)$ отлична от 0. Кроме того, если моном то пустой, то эта компонента является приведенным слева (справа) полиномом.

ДокАЗАТЕЛЬСтво. Будем считать, что $f=\alpha m_{0} x^{k} y u+m_{0} y g$. Второй случай рассматривается аналогично. На пространстве полиномов от переменных $x$ и $y$ определим линейные операторы $P_{0}$ и $P_{1}$, сопоставляющие каждому полиному его однородные компоненты степеней 0 и 1 по $x$ соответственно. Нетрудно проверить, что

$$
P_{1}(g \cdot h)=P_{0}(g) P_{1}(h)+P_{1}(g) P_{0}(h),
$$

где $g$ и $h$ - полиномы от $x$ и $y$. Мы хотим показать, что $P_{1}(f(x+y, y)) \neq 0$. Положим $m_{0}^{\prime}=m_{0}(x+y, y), u^{\prime}=u(x+y, y), g^{\prime}=g(x+y, y)$. Пользуясь равенством $(1)$, получаем

$$
\begin{gathered}
P_{1}(f(x+y, y))=P_{0}\left(m_{0}^{\prime}\right) P_{1}\left(\alpha(x+y)^{k} y u^{\prime}+y g^{\prime}\right)+P_{1}\left(m_{0}^{\prime}\right) P_{0}\left(\alpha(x+y)^{k} y u^{\prime}+y g^{\prime}\right) \\
=y^{s}\left(\alpha P_{0}\left((x+y)^{k}\right) y P_{1}\left(u^{\prime}\right)+\alpha P_{1}\left((x+y)^{k}\right) y P_{0}\left(u^{\prime}\right)+y P_{1}\left(g^{\prime}\right)\right)+P_{1}\left(m_{0}^{\prime}\right) \cdot 0
\end{gathered}
$$

Здесь $P_{0}\left(m_{0}^{\prime}\right)=y^{s}, s=\operatorname{deg} m_{0}$, так как $m_{0}$ - моном, а в силу полиоднородности $\alpha x^{k} y u+y g$ и равенства 0 суммы коэффициентов этого полинома

$$
P_{0}\left(\alpha(x+y)^{k} y u^{\prime}+y g^{\prime}\right)=0
$$

Поэтому

$$
\begin{aligned}
P_{1}(f(x+y, y)) & =y^{s}\left(\alpha y^{k+1} P_{1}\left(u^{\prime}\right)+\alpha\left(\sum_{i=0}^{k-1} y^{i} x y^{k-1-i}\right) y P_{0}\left(u^{\prime}\right)+y P_{1}\left(g^{\prime}\right)\right) \\
& =y^{s}\left(\alpha x y^{k-1} y P_{0}\left(u^{\prime}\right)+y(\cdot)\right) \neq 0,
\end{aligned}
$$

так как $P_{0}\left(u^{\prime}\right)$ - моном (возможно пустой). Полином $P_{1}(f(x+y, y))$ имеет по крайней мере два ненулевых коэффициента, и в случае $s=0$ он является приведенным слева. Лемма доказана. 
ДокаЗАТЕЛЬСТво тЕОРЕмЫ. 1) $\Longrightarrow 2$ ). Пусть алгебра $A$ удовлетворяет трехчленному тождеству $f=0$ степени $s$. Если полином $f$ имеет ненулевую сумму коэффициентов, то в алгебре $A$ вьполнено тождество $x^{s}=0$, из которого следует энгелевость степени $2 s-1$.

Если же сумма коэффициентов полинома $f$ нулевая, то в силу леммы 1 можно считать, что $f$ зависит только от двух переменных $x$ и $y$ и является по ним однородным. Далее воспользуемся леммой 2 , согласно которой в силу бесконечности поля $F$ в алгебре $A$ выполнено тождество

$$
y^{m}\left(\sum_{i=0}^{r} \alpha_{i} y^{i} x y^{r-i}\right) y^{k}=0,
$$

где $\alpha_{i} \in F, \alpha_{0} \neq 0, \alpha_{n} \neq 0, m+r+k=s$. При этом $m=0(k=0)$ в случае левой (правой) приведенности полинома $f$. В случае двусторонней приведенности $m=0$ и $k=0$. В работе [2] было показано, что из (2) следует тождество $y^{m} E_{3 r-1} y^{k}=0$.

$2) \Longrightarrow 3$ ). Допустим сначала, что поле $F$ имеет характеристику $p>0$. Пусть $s$ - наименьшее натуральное число со свойством $p^{s} \geqslant n$. Подставляя в тождество $y^{m} E_{n}(x, y) y^{k}=0 \quad x=E_{p^{s}-n}(x, y)$, получаем полугрупповое тождество

$$
y^{m} E_{p^{s}}(x, y) y^{k}=y^{m}\left(x y^{p^{s}}-y^{p^{s}} x\right) y^{k}=0 .
$$

Если же поле $F$ имеет нулевую характеристику, то зафиксируем целые неотрицательные числа $n>1, k, m$ (случай $n=1$ очевиден, и мы исключим его из дальнейшего рассмотрения) и обозначим через $T$ вербальньй идеал свободной ассоциативной алгебры счетного ранга $F\langle X\rangle, X=\left\{x, z, y=y_{0}, y_{1}, y_{2}, \ldots\right\}$, порожденный полиномом $y^{m} E_{n}(x, y) y^{k}$. Без ограничения обшности можно считать, что $n \geqslant m+k+1$. Рассмотрим идеал $I$ алгебры $F\langle X\rangle$, порожденный всеми элементами вида $\left[x, z, y_{i_{1}}, \ldots, y_{i_{l}}\right.$, $\left.\left[y_{i_{l+1}}, y_{i_{l+2}}\right], y_{i_{l+3}}, \ldots\right]$, где $y_{i_{j}} \in\left\{y_{0}=y, y_{1}, \ldots\right\}$. Покажем, что при $l=m+n+k$ имеет место сравнение

$$
y^{m}\left[x, y_{1}, \ldots, y_{l}, y\right] y^{k} \equiv 0(\bmod (T+I)) .
$$

При $i=0,1, \ldots, m+k$ рассмотрим полиномы $f_{i}\left(x, z, y, y_{1}, \ldots, y_{n-1+i}\right)$ степени $m+k+1$ по $y$, являющиеся частичными линеаризациями полиномов $y^{m} E_{n+i}([x, z], y) y^{k}$. Каждый полином $f_{i}$ есть сумма всевозможных слагаемых степени $m+k+1$ по $y=y_{0}$ и степени 1 по $y_{1}, \ldots, y_{n-1+i}$ вида

$$
y_{\alpha_{1}} \cdots y_{\alpha_{m}}\left[x, z, y_{\beta_{1}}, \ldots, y_{\beta_{n+i}}\right] y_{\gamma_{1}} \cdots y_{\gamma_{k}}
$$

По модулю идеала $I$ мы можем переставлять буквы в коммутаторах начиная с третьего места; этим мы постоянно будем пользоваться ниже. Обозначим через $f_{i}^{(s)}, s=1, \ldots$, $m+k+1$, сумму слагаемых вида (4), для которых $\beta_{1} \leqslant \beta_{2} \leqslant \cdots \leqslant \beta_{n+i}$ и среди $\beta_{j}$ нуль встречается $s$ раз. По модулю идеала $T+I$ имеем

$$
0 \equiv f_{i} \equiv \sum_{s=1}^{m+k+1} \frac{(n+i) !}{s !} f_{i}^{(s)}(\bmod (T+I)) .
$$


Для $i<j \leqslant m+k+1$ обозначим через $\Lambda_{i}^{j}$ следующее подмножество симметрической группы $\operatorname{Sym}(1,2, \ldots, n-1+j)$ :

$$
\Lambda_{i}^{j}=\left\{\left(\mu_{1}, \ldots, \mu_{j-i}, \lambda_{1}, \ldots, \lambda_{n-1+i}\right) \mid \mu_{1}<\cdots<\mu_{j-i}, \lambda_{1}<\cdots<\lambda_{n-1+i}\right\} .
$$

Если $\lambda \in \Lambda_{i}^{j}$ и

$$
f=\sum_{s=1}^{m+k+1} \alpha_{s} f_{i}^{(s)}\left(x, z, y, y_{1}, \ldots, y_{n-1+i}\right)
$$

то положим

$$
f^{\lambda}=f\left(\left[x, z, y_{\mu_{1}}, \ldots, y_{\mu_{j-i-1}}\right], y_{\mu_{j-i}}, y, y_{\lambda_{1}}, \ldots, y_{\lambda_{n-1+i}}\right) .
$$

Нетрудно проверить, что по модулю идеала $I$ имеет место равенство

$$
\sum_{\lambda \in \Lambda_{i}^{j}} f^{(\lambda)} \equiv \sum_{s=1}^{m+k+1} \alpha_{s} C_{n+j-s}^{j-i} f_{j}^{(s)}(\bmod I)
$$

Полагая $\beta_{s, i}^{(0)}=(m+k+1) ! / s !$, из (5) получаем

$$
0 \equiv f_{i} \equiv \sum_{s=1}^{m+k+1} \beta_{s, i}^{0} f_{i}^{(s)}(\bmod (T+I)), \quad i=0,1, \ldots, m+k
$$

Используя (6) при $f=f_{0}, j=i, i=0$ и (7), имеем

$$
\begin{aligned}
0 & \equiv C_{n+i-(m+k+1-0)}^{i-0} f_{i}-\sum_{\lambda \in \Lambda_{0}^{i}} f_{0}^{(\lambda)} \\
& \equiv \sum_{s=1}^{m+k} \beta_{s, i}^{(0)}\left(C_{n+i-(m+k+1-0)}^{i-0}-C_{n+i-s}^{i-0}\right) f_{i}^{(s)}(\bmod (T+I)), \quad i=1, \ldots, m+k .
\end{aligned}
$$

Полагая $\beta_{s, i}^{(t+1)}=\beta_{s, i}^{(t)}\left(C_{n+i-(m+k+1-t)}^{i-t}-C_{n+i-s}^{i-t}\right)$ и действуя по индукции аналогично изложенному выше, мы в итоге получим

$$
\beta_{1, m+k}^{(m+k)} f_{m+k}^{(1)} \equiv 0(\bmod (T+I))
$$

откуда в силу неравенства $\beta_{1, m+k}^{(m+k)} \neq 0$ сразу следует (3).

Для $i=1,2, \ldots$ и $j=0,1, \ldots, l$ положим $y_{j}^{(i)}=y_{(l+1) i+j}$. Определим последовательность полиномов $F^{(i)}$ (при фиксированных $m, l, k$ ):

$$
\begin{gathered}
F^{(0)}=[x, y], \ldots, \\
F^{(i+1)}=\left(y_{0}^{(i+1)}\right)^{m}\left[F^{(i)}, y_{1}^{(i+1)}, \ldots, y_{l}^{(i+1)}, y_{0}^{(i+1)}\right]\left(y_{0}^{(i+1)}\right)^{k} .
\end{gathered}
$$

Пусть $B$ есть относительно свободная двупорожденная алгебра нематричного многообразия $\operatorname{Var}(A)$. Из теоремы Кемера [3] о нильпотентности радикала Джекобсона 
конечнопорожденной $P I$-алгебры над полем нулевой характеристики следует, что при некотором $d$ алгебра $B$ удовлетворяет всем тождествам вида

$$
z_{1}\left[x_{1}, x_{2}\right] z_{2} \cdots z_{d}\left[x_{2 d-1}, x_{2 d}\right] z_{d+1}=0, \quad x_{i} \in F\langle X\rangle, \quad z_{i} \in F\langle X\rangle \cup\{1\}
$$

Из равенства (3) и определения полиномов $F^{(i)}=0$ получаем

$$
F^{(d)} \equiv \sum g_{1} w_{1} g_{2} w_{2} \cdots g_{d} w_{d} g_{d+1}(\bmod T), \quad g_{i} \in F\langle X\rangle \cup\{1\}, \quad w_{i}=\left[y_{\alpha}^{(j)}, y_{\beta}^{(j)}\right]
$$

В совокупности с (8) равенство (9) означает, что в алгебре $B$ вьполнено тождество $F^{(d)}=0$ (при $m=k=0$ в точности получается лиева нильпотентность).

Для доказательства импликации достаточно установить, что тождество $F^{(d)}=0$ влечет полугрупповое тождество от двух переменных, приведенное (приведенное слева, приведенное справа) при $m=k=0(m=0, k=0)$. Допустим, что при некоторой подстановке переменных в $F^{(i)}$ мы получили полином в форме $\left[a_{i}, b_{i}\right]$, где $a_{i}$ и $b_{i}$ - мономы от $x$ и $y$. Подставляя вместо переменных $y_{1}^{(i+1)}, \ldots, y_{l}^{(i+1)}, y_{0}^{(l+1)}$ соответствующие куски слова Морса, построенного на переменных $a_{i}$ и $b_{i}$, мы получим некоторый полином от двух переменных вида $\left[a_{i+1}, b_{i+1}\right]$, где $a_{i+1}$ и $b_{i+1}-$ мономы. Для построенного таким образом тождества $\left[a_{d}, b_{d}\right]=0$ вьполняются требуемые свойства приведенности.

$3) \Longrightarrow 1)$. Тривиально.

$2) \Longrightarrow 4)$. Алгебра с единищей $\Lambda_{2}$ над бесконечным полем не удовлетворяет тождеству $y^{m} E_{n} y^{k}=0$. В самом деле, его следствием является тождество энгелевости $E_{n}=0$, подставляя в которое $x=e_{12}, y=e_{11}$ ( $e_{11}$ и $e_{12}$ - матричные "единички"), получаем $\pm e_{12}$.

$4) \Longrightarrow 2)$. Обозначим идеал тождеств алгебры $\Lambda_{2}$ через $T$. Пусть полином $f=f\left(x_{1}\right.$, $\left.\ldots, x_{n}\right)$ степени $m$ является тождеством алгебры $A$, но $f \notin T$. Если сумма коэффициентов полинома $f$ ненулевая или $m<3$, то доказьваемая импликация очевидна. Поэтому будем предполагать, что $f$ имеет нулевую сумму коэффициентов и $m \geqslant 3$.

Покажем, что при некотором $s A$ удовлетворяет нетривиальному тождеству вида

$$
\sum_{i=0}^{s} \alpha_{i} y^{i} x y^{s-i}=0
$$

При доказательстве импликации 1) $\Longrightarrow 2$ ) мы уже видели, что такое тождество влечет при некоторых $m, l, k$ тождество $y^{m} E_{l}(x, y) y^{k}=0$.

Представим $f$ в виде линейной комбинации полиномов вида

$$
\left[x_{i_{1}}, x_{i_{2}}, x_{i_{3}}, \ldots, x_{i_{k}}\right] x_{i_{k+1}} \cdots x_{i_{m}}, \quad k \geqslant 2 \text {. }
$$

Полином вида $(11)$ назовем правильным м, если $i_{1} \leqslant i_{r}, r=2, \ldots, k, i_{1} \neq i_{2}, i_{3} \leqslant \cdots \leqslant i_{k}$ и $i_{k+1} \leqslant \cdots \leqslant i_{m}$. Любой полином вида (11) представи́м как линейная комбинация правильного полинома и полиномов вида

$$
g_{1}\left[x_{\alpha}, x_{\beta}\right] g_{2}\left[x_{\gamma}, x_{\delta}\right] g_{3}, \quad g_{i} \in F\langle X\rangle \cup\{1\}
$$


Производя перенумерацию переменных и учитьвая, что $f \notin T$, представим $f$ в виде $f=f_{1}+f_{2}+f_{3}+g$, где $g$ - сумма слагаемых вида (12), $f_{1}$ - сумма правильных полиномов с $i_{1}=1, i_{2}=2, f_{2}$ - сумма правильных полиномов с $i_{1}=2, f_{3}$ - сумма прочих правильных полиномов и в $f_{1}$ хотя бы одно слагаемое входит с ненулевым коэффициентом. Подставим в $f x_{2}=x, x_{i}=y^{l_{i}}$ при $i \neq 2$ (выбор натуральных чисел $l_{i}$ осуществим позднее). При такой подстановке $f_{3}$ перейдет в 0, а $f_{1}, f_{2}, g$ перейдут в $f_{1}^{\prime}, f_{2}^{\prime}, g^{\prime}$ соответственно. В полученном полиноме сделаем подстановку $x=x+y$ и выделим однородную компоненту степени 1 по $x$. Вклад в нее полинома $g^{\prime}$ будет нулевым, так как на два коммутатора приходится только один $x$. Вычислим вклад полиномов $f_{1}^{\prime}, f_{2}^{\prime}$.

Пусть $\operatorname{deg}_{x_{i}} f=k_{i}>0$. Обозначим через $\Lambda_{1}$ множество полиномов вида

$$
[y^{l_{1}}, x, \underbrace{y^{l_{1}}, \ldots, y^{l_{1}}}_{\alpha_{1}}, \underbrace{y, \ldots, y}_{\alpha_{2}}, \underbrace{y^{l_{3}}, \ldots, y^{l_{3}}}_{\alpha_{3}}, \ldots]\left(y^{l_{1}}\right)^{\beta_{1}}(y)^{\beta_{2}}\left(y^{l_{3}}\right)^{\beta_{3}} \cdots\left(y^{l_{n}}\right)^{\beta_{n}},
$$

где $\alpha_{j}, \beta_{j} \geqslant 0,1+\alpha_{1}+\beta_{1}=k_{1}, 1+\alpha_{2}+\beta_{2}=k_{2}, \alpha_{i}+\beta_{i}=k_{i}$ при $i>2$. Обозначим через $\Lambda_{2}$ множество полиномов вида

$$
[x, y^{l_{i}}, \underbrace{y, \ldots, y}_{\alpha_{2}}, \underbrace{y^{l_{3}}, \ldots, y^{l_{3}}}_{\alpha_{3}}, \ldots]\left(y^{l_{1}}\right)^{k_{1}}(y)^{\beta_{2}}\left(y^{l_{3}}\right)^{\beta_{3}} \cdots\left(y^{l_{n}}\right)^{\beta_{n}}
$$

где $\alpha_{j}, \beta_{j} \geqslant 0,1+\alpha_{i}+\beta_{i}=k_{i}$ при $i \geqslant 2$.

Очевидно, что вкладполиномов $f_{1}^{\prime}, f_{2}^{\prime}$ в вычисляемую компоненту степени 1 по $x$ представляет собой линейную комбинацию полиномов из $\Lambda_{1}$ и $\Lambda_{2}$ соответственно. В силу построения $f_{1}$ один из коэффициентов этой комбинации отличен от 0. Определим переменные $l_{i}$ так, чтобы полиномы из $\Lambda_{1} \cup \Lambda_{2}$ были линейно независимыми. Тогда $A$ будет удовлетворять некоторому нетривиальному тождеству вида (10), что и доказьвает теорему.

Автор, пользуясь случаем, выражает глубокую признательность своему научному руководителю А.Р. Кемеру за постановку задачи и внимание к работе.

\section{СПИСОК ЦИТИРОВАННОЙ ЛИТЕРАТУРЫ}

[1] Голубчик И.З., Михалёв А. В. О многообразиях алгебр с полугрупповым тождеством // Вестн. МГУ. Сер. 1. Матем., мех. 1982. № 2. С. 8-11.

[2] Riley D. M., Wilson M. C. Associative Algebras Satisfying a Semigroup Identity. Preprint, 1997.

[3] Кемер А.Р. Тождества Капелли и нильпотентность радикала Джекобсона конечнопорожденной $P I$-алгебры // Докл. АН СССР. 1980. Т. 225. № 4. С. 793-797.

Филиал Московского государственного университета им. М. В. Ломоносова, г. Ульяновск

Поступило

E-mail: samoilov@mmf.univ.simbirsk.su 03.02.98 